\title{
Modeling and Analysis of Magnetic Nanoparticles Injection in Water-Oil Two-Phase Flow in Porous Media under Magnetic Field Effect
}

\author{
Mohamed F. El-Amin, ${ }^{1,2,3}$ Ahmed M. Saad, ${ }^{2}$ Amgad Salama, ${ }^{4}$ and Shuyu Sun ${ }^{2}$ \\ ${ }^{1}$ College of Engineering, Effat University, Jeddah 21478, Saudi Arabia \\ ${ }^{2}$ Computational Transport Phenomena Laboratory (CTPL), Division of Physical Sciences and Engineering (PSE), \\ King Abdullah University of Science and Technology (KAUST), Thuwal, Jeddah 23955-6900, Saudi Arabia \\ ${ }^{3}$ Mathematics Department, Faculty of Science, Aswan University, Aswan 81528, Egypt \\ ${ }^{4}$ Faculty of Engineering, University of Regina, Regina, SK, Canada
}

Correspondence should be addressed to Mohamed F. El-Amin; mohamed.elamin.kaust@gmail.com

Received 23 February 2017; Revised 20 June 2017; Accepted 26 July 2017; Published 28 August 2017

Academic Editor: Timothy S. Collett

Copyright (c) 2017 Mohamed F. El-Amin et al. This is an open access article distributed under the Creative Commons Attribution License, which permits unrestricted use, distribution, and reproduction in any medium, provided the original work is properly cited.

\begin{abstract}
In this paper, the magnetic nanoparticles are injected into a water-oil, two-phase system under the influence of an external permanent magnetic field. We lay down the mathematical model and provide a set of numerical exercises of hypothetical cases to show how an external magnetic field can influence the transport of nanoparticles in the proposed two-phase system in porous media. We treat the water-nanoparticles suspension as a miscible mixture, whereas it is immiscible with the oil phase. The magnetization properties, the density, and the viscosity of the ferrofluids are obtained based on mixture theory relationships. In the mathematical model, the phase pressure contains additional term to account for the extra pressures due to fluid magnetization effect and the magnetostrictive effect. As a proof of concept, the proposed model is applied on a countercurrent imbibition flow system in which both the displacing and the displaced fluids move in opposite directions. Physical variables, including waternanoparticles suspension saturation, nanoparticles concentration, and pore wall/throat concentrations of deposited nanoparticles, are investigated under the influence of the magnetic field. Two different locations of the magnet are studied numerically, and variations in permeability and porosity are considered.
\end{abstract}

\section{Introduction}

Industry is now looking seriously into using nanotechnology as a viable tool to solving new challenges in several fields. In particular, there has been interest among oil and gas production companies to explore using nanotechnology in solving challenges related to unconventional oil and gas reservoirs, such as those found in tight and shale formations [1-7]. Nanotechnology has been used in different areas of the oil and gas industry from exploration, drilling, production to reservoir monitoring, and refining. The conventional Enhanced Oil Recovery (EOR) methods have several problems from high cost to low oil recovery in addition to operations problems especially in thermal and chemical methods. The nature of nanoparticles results in some useful characteristics such as increased surface area, which at the nanoscale size, does matter when it comes to how molecules react to and bond with each other. So, for example, nanoparticles can be used in EOR, because they are small enough to pass through pore throats in typical reservoirs, and they can be retained by the rock. Ju and Fan [8] calibrated a model for nanoparticles transport in two-phase flow in porous media based on the formulation of the colloid model of fine particles transport in two-phase flow in porous media [9]. El-Amin et al. [1013] have presented modeling and simulations of nanoparticles transport associated with two-phase flow in porous media. On the other hand, experimental studies of using nanoparticles in EOR have been conducted by Suleimanov et al. [14] and Hendraningrat et al. [15]. 
One of the prospective applications of nanotechnology is nanoferrofluids, as the flow of such fluids can be controlled by the introduction of external magnetic field. This opens the way for various applications from directing flow in reservoir monitoring, diverting flow in acid jobs to control and boost of injection fluid advancement during pressure maintenance to increase oil recovery. The idea of using a strong external magnetic field with large magnetic susceptibility fluid is to mobilize ferrofluid through porous media. Oil recovery can be increased by using nanoparticles with electromagnetic properties (such as iron oxide, $\mathrm{Fe}_{2} \mathrm{O}_{3}$, and zinc oxide, $\mathrm{ZnO}$ ) under waves generated from an electromagnetic source. Both direct and alternating magnetic fields are under investigation; in our case here we will focus on direct magnetic field. The magnetization of the particles and their attraction toward the magnet causes flow of the magnetic particles suspension. The movement of the magnetic nanoparticles under the magnetic field effect is independent of the orientation of the magnet. In the last few years, a number of publications have been considered nanoferrofluids in oil and gas recovery or environmental applications (e.g., [16-23]).

Borglin et al. [24] conducted experiments to measure the magnetic induction, which converted to magnetic field strength, at various distances in a direction aligned with the poles. McCaig and Clegg [25] presented equations which indicate that ferrofluid magnetization is variable at all locations far away from the magnet due to the decreasing magnetic field strength. The gradient of magnetic field strength varies also with distance from the magnet. Moreover, the force on the ferrofluid decreases with distance from a magnet. Therefore, we may conclude that the distance from the magnet is important and should be reconsidered with taking into consideration its location in particular in the core-scale as well as the direction of flow. On the other hand, it is known that, in the presence of an external magnetic field, the nanoparticles in ferrofluid become magnetized and are pulled toward a magnet. In this work, we attempt to discuss the effect of location of the magnet on the transport of the nanoparticles in porous media.

In the current work, we develop a mathematical model to describe the magnetic nanoparticles-water suspension imbibition into an initially oil saturated porous domain under magnetic field effect. The porous medium is considered initially saturated totally with oil except for a residual amount of the other phase. We consider countercurrent imbibition into a small-scale porous core. This countercurrent imbibition refers to the case in which all the porous medium domain boundaries have no flow except one side. Physical variables are investigated under the influences of magnetic field with two different locations of magnet, namely, right and left to the porous core. Numerical experiments for the two cases are performed and results are introduced in graphical representations.

\section{Modeling and Mathematical Formulation}

Consider suspension of magnetic nanoparticles injected in an isothermal incompressible water-oil two-phase flow under an external magnetic field. In the following, we describe the mathematical modeling of the problem under consideration.

In the following subsections, we firstly introduce the magnetic force and other magnetic modeling. The magnetic body force, which acts as a body force on the nanoparticles suspension per unit volume, appears in the extended Darcy's law as presented in the second subsection. Other magnetic parameters such as magnetization and magnetic field strength are also presented in the first subsection. Then, the governing flow equations such as momentum (extended Darcy's law) and mass conservation (saturation equation) are provided in the second subsection. The third subsection is devoted to the modeling of nanoparticles transport which is coupled with the flow equations via velocity and saturation. Finally, the initial and boundary conditions are presented in the last subsection.

2.1. Magnetic Force. The magnetization (polarization) of the nanoparticles suspension interacts with the external permanent magnetic field to produce attractive forces on each particle. The external magnetic force acts as a body force on the nanoparticles suspension per unit volume which can be expressed as [16]

$$
\mathbf{F}_{\text {mag }}=\mu_{0} M \frac{\partial H}{\partial z},
$$

where $\mu_{0}$ is the magnetic permeability, $M$ is the magnetization, and $H$ is the magnetic field strength. The magnetization $M$ is a function of $H$, approximated by

$$
M=a_{1} \tan ^{-1}\left(b_{1} H\right),
$$

where the parameters $a_{1}$ and $b_{1}$ depend on the particular type of the ferromagnetic material. The values of the initial susceptibility and the saturation magnetization are controlled by the parameters $a_{1}$ and $b_{1}$, respectively. The larger $b_{1}$ leads to a larger initial susceptibility which corresponds to larger particles or agglomeration of particles. The range of $a_{1}$ may be of order $10^{4}-10^{5}$, while the order of $b_{1}$ may be of order $10^{-6}-10^{-5}$. The magnetic field strength in $1 \mathrm{D}$ may be written as follows [16]:

$$
\begin{aligned}
H_{z} & =\frac{B_{r}}{\pi \mu_{0}}\left(\tan ^{-1} \frac{a b}{z\left(a^{2}+b^{2}+z^{2}\right)^{1 / 2}}\right. \\
& \left.-\tan ^{-1} \frac{a b}{(z+L)\left(a^{2}+b^{2}+(z+L)^{2}\right)^{1 / 2}}\right),
\end{aligned}
$$

where $B_{r}$ is the residual magnetization (in this work, $B_{r}=$ $1.19[\mathrm{~T}])$ and $L$ is the distance between the poles of the magnet. In Figure $1, F_{\text {mag }}, H_{z}$, and $\partial H_{z} / \partial z$ are plotted against $z$.

Oldenburg et al. [16] reported that magnetic particles (ferrofluids) could have both miscible and immiscible behaviors with water. For example, an oil-based ferrofluid is immiscible with water while aqueous ferrofluids are miscible in water. The immiscible case should be described by twophase aqueous/nonaqueous mixtures regardless of external 


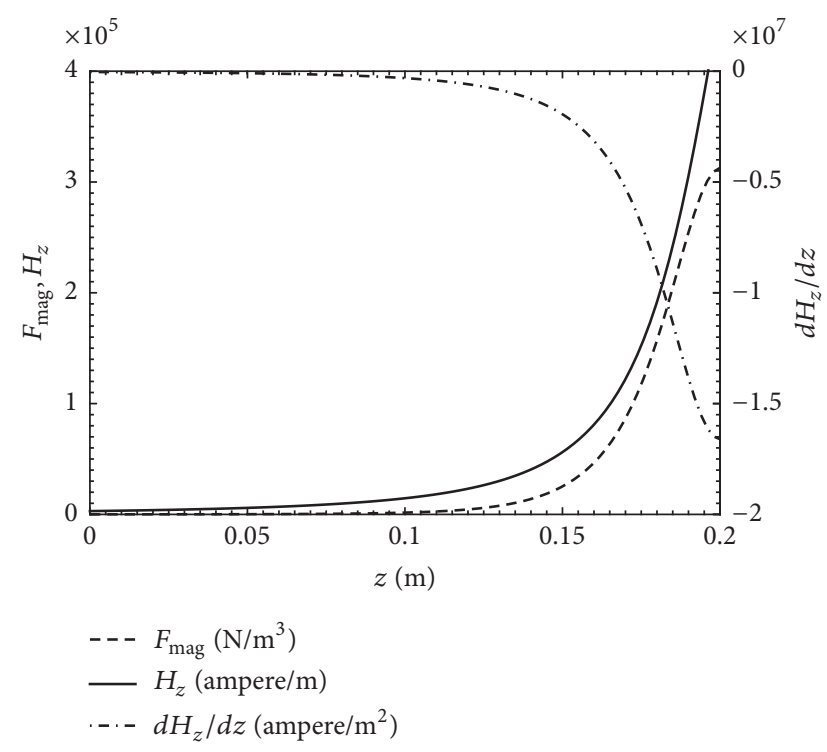

FIGURE 1: $F_{\text {mag }}, H_{z}$, and $\partial H_{z} / \partial z$ are plotted against $z$.

magnetic field strength, while the case of miscible in water should be described as single-phase aqueous mixtures. Under no external magnetic field, the two fluids are miscible. When a magnetic field is applied, it affects the ferrofluid part of the aquatic mixture in such a way that it behaves as immiscible. That is, the ferrofluids motion is dictated by the magnetic field. When the magnetic field is relatively strong, its effect on the ferrofluids may be influential. Therefore, a two-phase flow model is suitable for the flow of ferrofluids under strong external magnetic fields, but when the magnet is at larger distances from the flow (thus segregation effects are unimportant), the fully miscible single-phase liquid model is appropriate. Both of these models assume that the mixture is not so dilute. Pure ferrofluid and dilute colloidal suspension have approximately a thousand particles per cubic meter (see $[16,26])$. So, one may conclude that the miscible and immiscible models may be applicable for ferrofluid fractions of $10 \%$ in water, and they are probably satisfactory in much more dilute mixtures [16].

In the current model, we treat the water-nanoparticles suspension as a miscible mixture while it is immiscible with the oil phase. Oldenburg et al. [16] assumed that magnetization increases linearly with the ferrofluid mass fraction $c$,

$$
M(c)=M(c=1) c .
$$

In the case of the immiscible two-phase (water-oil) model, Oldenburg et al. [16] assumed that magnetization increases linearly with the ferrofluid saturation,

$$
M\left(S_{w}\right)=M\left(S_{w}=1\right) S_{w} .
$$

For the immiscible conceptualization, density and viscosity are considered properties of the respective phases, and no mixing relations are required. Therefore, in the current model we consider immiscible two-phase (water-oil) model; however, the water-nanoparticles suspension is a miscible mixture. So, one may expresses the the magnetization of this system as,

$$
M\left(S_{w}, c\right)=M\left(S_{w}=1, c=1\right) S_{w} c .
$$

Following the work of Reeves and Cranwell [27] and Herbert et al. [28] on brine-water mixtures, Oldenburg et al. [16] have assumed that the volumes of pure water and ferrofluid are additive and define for the mixture density as

$$
\frac{1}{\rho_{w}}=\frac{1-c}{\rho_{w, p}}+\frac{c}{\rho_{f}},
$$

where $\rho_{w, p}\left[\mathrm{~kg} \cdot \mathrm{m}^{-3}\right]$ is the density of water component and $\rho_{f}\left[\mathrm{~kg} \cdot \mathrm{m}^{-3}\right]$ is the density of ferrofluid component.

The viscosity of the nanoparticles-water mixture is calculated by the following linear relationship $[16,28]$ :

$$
\mu_{w}=\mu_{w, p}(1+1.35 c),
$$

where $\mu_{w, p}$ is the viscosity of pure water.

2.2. Flow Model. Considering the external magnetic force, which acts as a body force as explained in the above subsection, the vertical $1 \mathrm{D}$ (i.e., $z$-direction) velocities of oil phase and nanoparticles-water mixture phase may be written as

$$
\begin{aligned}
& u_{o}=-\frac{K k_{r o}}{\mu_{o}}\left(\frac{\partial p_{o}}{\partial z}-\rho_{o} g\right), \\
& u_{w}=-\frac{K k_{r w}}{\mu_{w}}\left(\frac{\partial p_{w}}{\partial z}-\rho_{w}(c) g-\mu_{0} M\left(S_{w}, c\right) \frac{\partial H}{\partial z}\right) .
\end{aligned}
$$

The mass conservation equation is

$$
\phi \frac{\partial S_{\alpha}}{\partial t}+\frac{\partial u_{\alpha}}{\partial z}=0, \quad \alpha=w, o,
$$

where $\phi[-]$ is the porosity, $\rho_{\alpha}\left[\mathrm{kg} \cdot \mathrm{m}^{-3}\right]$ is the density of phase $\alpha, S_{\alpha}[-]$ is the saturation of phase $\alpha$, and $\mathbf{u}_{\alpha}\left[\mathrm{m} \cdot \mathrm{s}^{-1}\right]$ is the velocity of the phase $\alpha . w$ stands for the nanoparticles-water suspension phase, and $o$ stands for the oil phase. $K\left[\mathrm{~m}^{2}\right]$ is the permeability, $k_{r \alpha}[-]$ is the relative permeability of phase $\alpha$, $p_{\alpha}[\mathrm{Pa}]$ is the pressure of phase $\alpha, g\left[\mathrm{~m} \cdot \mathrm{s}^{-2}\right]$ is the gravitational acceleration, and $\mu_{\alpha}\left[\mathrm{kg} \mathrm{m}^{-1} \mathrm{~s}^{-1}\right]$ is the viscosity of phase $\alpha$. The fluid saturations for the two-phase flow of water and oil are related by

$$
S_{w}+S_{o}=1
$$

In countercurrent imbibition, the sum of the velocities of the wetting and nonwetting phases is zero, that is,

$$
u_{t}=u_{w}+u_{o}=0
$$

The capillary pressure is defined as

$$
p_{c}=p_{o}-p_{w}
$$


Since, in this case of study, we have two phases, one magnetized and the other nonmagnetized, the magnetostrictive effect should not be neglected. The magnetized phase pressure will have additional pressure term with the conventional thermodynamic pressure. The resulting pressure is called composite pressure [21], which can be given as

$$
p_{w}^{*}=p_{w}+\left(p_{m}+p_{s}+p_{n}\right),
$$

where $p_{w}$ is the ferrofluid phase dynamic pressure, $p_{m}$ is the fluid magnetic pressure, $p_{s}$ is the magnetostrictive pressure, and $p_{n}$ is the magnetic normal pressure, which is neglected [21] in this study. Also, one can write

$$
\begin{aligned}
p_{c}^{*} & =p_{c}-\left(p_{m}+p_{s}\right), \\
p_{s} & =\mu_{0} \int_{0}^{H} V\left(\frac{\partial M}{\partial V}\right)_{H, T} d H, \\
p_{m} & =\mu_{0} \int_{0}^{H} M d H .
\end{aligned}
$$

The specific volume is given as

$$
V=\partial\left(\frac{1}{\rho}\right)=-\frac{1}{\rho^{2}} \partial \rho,
$$

So,

$$
p_{s}=-\mu_{0} \int_{0}^{H} \rho\left(\frac{\partial M}{\partial \rho}\right)_{H, T} d H
$$

where

$$
\begin{aligned}
\frac{\partial M}{\partial \rho}= & \left(\frac{\partial M}{\partial a_{1}}\right)_{b_{1}, H}\left(\frac{\partial a_{1}}{\partial \rho}\right)+\left(\frac{\partial M}{\partial b_{1}}\right)_{a_{1}, H}\left(\frac{\partial b_{1}}{\partial \rho}\right) \\
& +\left(\frac{\partial M}{\partial H}\right)_{a_{1}, b_{1}}\left(\frac{\partial H}{\partial \rho}\right) .
\end{aligned}
$$

We may approximate $\partial a_{1} / \partial \rho$ and $\partial b_{1} / \partial \rho$, when $a_{1, o}$ and $b_{1, o}$ are equal to zero in the nonmagnetized phase, as

$$
\begin{aligned}
& \frac{\partial a_{1}}{\partial \rho} \approx \frac{a_{1}-a_{1, o}}{\rho_{w}-\rho_{o}}=\frac{a_{1}}{\rho_{w}-\rho_{o}}, \\
& \frac{\partial b_{1}}{\partial \rho} \approx \frac{b_{1}-b_{1, o}}{\rho_{w}-\rho_{o}}=\frac{a_{1}}{\rho_{w}-\rho_{o}} .
\end{aligned}
$$

Therefore,

$$
p_{\mathrm{s}}=\frac{\rho_{w} a_{1}}{\rho_{w}-\rho_{o}} \mu_{0} H \tan ^{-1}\left(b_{1} H\right) .
$$

Similarly, therefore,

$$
p_{m}=a_{1}\left(H \tan ^{-1}\left(b_{1} H\right)-\frac{1}{2 b_{1}} \ln \left(b_{1}^{2} H^{2}+1\right)\right) .
$$

Substituting from (9), (13), (20), and (21) into (12) and eliminating $\partial p_{o} / \partial z$, we may have

$$
\begin{aligned}
\frac{\partial p_{w}^{*}}{\partial z}= & -f_{w} \frac{\partial p_{c}^{*}}{\partial z}+\left(\rho_{w} f_{w}+\rho_{o} f_{o}\right) g \\
& +f_{w} \mu_{0} M\left(S_{w}, c\right) \frac{\partial H}{\partial z},
\end{aligned}
$$

So, the water velocity becomes

$$
u_{w}=K \lambda_{w} f_{o}\left(\frac{\partial p_{c}^{*}}{\partial z}-\Delta \rho g+\mu_{0} M\left(S_{w}, c\right) \frac{\partial H}{\partial z}\right) .
$$

Therefore, the saturation equation for the water phase becomes

$$
\begin{aligned}
& \phi \frac{\partial S_{w}}{\partial t} \\
& \quad+\frac{\partial}{\partial z}\left[K \lambda_{w} f_{o}\left(\frac{\partial p_{c}^{*}}{\partial z}-\Delta \rho g+\mu_{0} M\left(S_{w}, c\right) \frac{\partial H}{\partial z}\right)\right] \\
& \quad=0,
\end{aligned}
$$

where $\lambda_{w}=k_{r w} / \mu_{w}$ and $\lambda_{o}=k_{r o} / \mu_{o}$ are mobility ratios of water and oil phases, respectively. $\lambda_{t}=\lambda_{w}+\lambda_{o}$ is the total mobility. $f_{w}=\lambda_{w} / \lambda_{t}$ and $f_{o}=\lambda_{o} / \lambda_{t}$ are the flow fraction of water and oil phases, respectively. $\Delta \rho=\rho_{w}-\rho_{o}$. The capillary pressure is a function of the normalized saturation which can be given as [29]

$$
p_{c}=p_{d} \ln S,
$$

where $p_{d}$ is the entry pressure for the imbibition. Moreover, the relative permeabilities are defined as

$$
\begin{aligned}
& k_{r w}=k_{r w}^{0} S^{a_{2}}, \\
& k_{r o}=k_{r o}^{0}(1-S)^{b_{2},}
\end{aligned}
$$

where $S$ is the normalized water phase saturation, which is given as

$$
S=\frac{S_{w}-S_{i w}}{1-S_{r o}-S_{i w}}, \quad 0<S<1,
$$

and $k_{r w}^{0}=k_{r w}(S=1)$ and $k_{r o}^{0}=k_{r o}(S=0)$ are the endpoint relative permeability of the water and oil phase, respectively. $a_{2}$ and $b_{2}$ are positive numbers. $S_{i w}$ is the irreducible water saturation and $S_{r o}$ is the residual oil saturation.

2.3. Nanoparticles Transport Model. Assuming that the nanoparticles exist only in the water phase and have only one size interval. The Brownian diffusion is considered for the nanoparticles and the volumes of pure water and the particles suspension are additive. The transport equation of the nanoparticles-water suspension in the water phase can be written as

$$
\begin{aligned}
& \phi \frac{\partial\left(S_{w} c\right)}{\partial t}-\frac{\partial c_{s 1}}{\partial t}-\frac{\partial c_{s 2}}{\partial t} \\
& \quad+\frac{\partial}{\partial z}\left(u_{w} c-\phi S_{w}\left(D_{\text {diff }}+D_{\text {disp }}\right) \frac{\partial c}{\partial z}\right)=0
\end{aligned}
$$

where $c\left[\mathrm{~m}^{3} \cdot \mathrm{m}^{-3}\right]$ is the volume concentration of nanoparticles in the water phase. $c_{s 1}\left[\mathrm{~m}^{3} \cdot \mathrm{m}^{-3}\right]$ is the volume of the nanoparticles in contact with the water phase available on the pore surfaces per unit bulk volume of the porous medium. $c_{s 2}\left[\mathrm{~m}^{3} \cdot \mathrm{m}^{-3}\right]$ is the volume of the nanoparticles entrapped in 
pore throats from the water phase per unit bulk volume of porous medium due to plugging and bridging. $D_{\text {diff }}\left[\mathrm{m}^{2} \cdot \mathrm{s}^{-1}\right]$ is the molecular diffusion coefficient which can be calculated using the Stokes-Einstein equation,

$$
D_{\text {diff }}=\tau \frac{k_{B} T}{3 \pi \mu_{w} d}
$$

and $\tau$ is the tortuosity of the flow. $k_{B}$ is the Boltzmann constant; $T[\mathrm{~K}]$ absolute temperature. The mechanical dispersion coefficient $D_{\text {disp }}\left[\mathrm{m}^{2} \cdot \mathrm{s}^{-1}\right]$ is a function of Darcy's velocity and may be given as

$$
\phi S_{w} D_{\text {disp }}=d_{l, w}\left|u_{w}\right|,
$$

where $d_{l, w}$ is the longitudinal dispersion coefficient.

The modified Gruesbeck-Collins model [30] for the surface deposition is used in this study $[8,12]$. In the presence of a critical velocity of the surface deposition only particle retention occurs while above it retention and entrainment of the nanoparticles take place simultaneously, which can be modeled as

$$
\frac{\partial c_{s 1}}{\partial t}= \begin{cases}\gamma_{d}\left|u_{w}\right| c, & u_{w} \leq u_{c} \\ \gamma_{d}\left|u_{w}\right| c-\gamma_{e}\left|u_{w}-u_{c}\right| c_{s 1}, & u_{w}>u_{c} .\end{cases}
$$

Also, the rate of entrapment of the nanoparticles in the water phase is given by

$$
\frac{\partial c_{s 2}}{\partial t}=\gamma_{p t}\left|u_{w}\right| c
$$

where $\gamma_{d}\left[\mathrm{~m}^{-1}\right]$ is the rate coefficient for surface retention of the nanoparticles in the water phase. $\gamma_{e}\left[\mathrm{~m}^{-1}\right]$ is the rate coefficient for entrainment of the nanoparticles. $u_{c}$ is the critical velocity for the water phase. $\gamma_{p t}\left[\mathrm{~m}^{-1}\right]$ is the pore throat blocking constant.

The porosity variation due to nanoparticles deposition is given as $[8,9]$

$$
\phi=\phi_{0}-\delta \phi, \quad \delta \phi=c_{s 1}+c_{s 2}
$$

where $\phi_{0}$ is the initial porosity. Therefore, permeability can be changed as [8]

$$
K=K_{0}\left[(1-f) k_{f}+f \frac{\phi}{\phi_{0}}\right]^{l},
$$

where $K_{0}$ is the initial permeability and $K=k \mathbf{I}, K_{0}=k_{0} \mathbf{I}$, where $k\left[\mathrm{~m}^{2}\right]$ and $k_{0}\left[\mathrm{~m}^{2}\right]$ are positive constants and $\mathbf{I}$ is a unit matrix. $k_{f}$ is constant for fluid seepage allowed by the plugged pores. The flow efficiency factor expressing the fraction of unplugged pores available for flow is given by

$$
f=1-\gamma_{f} \mathcal{c}_{s 2},
$$

where $\gamma_{f}$ is the coefficient of flow efficiency for the nanoparticles. The exponent $l$ has a value of the range from 2.5 to 3.5.

The variation of relative permeabilities due to the nanoparticles reposition is given by El-Amin et al. [12] as follows:

$$
k_{r \alpha, p}=\left[1+r_{a}\left(\theta_{\alpha}-1\right)\right] k_{r \alpha}, \quad \alpha=w, o,
$$

where $\theta_{\alpha}$ is the ratio of the phase $\alpha$ relative permeability due to nanoparticles adhering; that is,

$$
k_{r \alpha, c}=\theta_{\alpha} k_{r \alpha}, \quad \alpha=w, o,
$$

where $k_{r \alpha, c}$ is the relative permeabilities of water/oil phase when the surfaces per unit bulk volume of the porous media is completely occupied by the nanoparticles. $r_{a}=a_{\mathrm{tot}} / a_{\mathrm{sp}}$ such that

$$
a_{\mathrm{sp}}=A \phi\left(\frac{\phi}{K}\right)^{1 / 2}
$$

is a specific area of the sand core and $A\left[\mathrm{~m}^{2}\right]$ is the crosssectional area. Also, the total surface area in contact with fluids per unit bulk volume is defined as [8]

$$
a_{\mathrm{tot}}=\frac{6 \beta}{d} \delta \phi
$$

and $d$ is the diameter of the nanoparticles in a given interval size.

2.4. Initial and Boundary Conditions. At the beginning of the flow the following initial conditions are considered:

$$
\begin{aligned}
S_{w} & =S_{w}^{0} \quad \text { at } t=0,0 \leq z \leq h, \\
c & =c_{s 1}=c_{s 2}=0 \text { at } t=0,0 \leq z \leq h,
\end{aligned}
$$

where $h$ is the rock depth and $S_{w}^{0}$ is the initial water saturation.

The boundary conditions are

$$
\begin{aligned}
S_{w} & =1-S_{o}^{0}, \\
c & =c_{0}, \\
c_{s 1} & =c_{s 2}=0
\end{aligned}
$$

$$
\text { at } t>0, z=0 \text {, }
$$

$$
\frac{d S_{w}}{d z}=\frac{d c}{d z}=\frac{d c_{s 1}}{d z}=\frac{d c_{s 2}}{d z}=0 \quad \text { at } t>0, z=h,
$$

where $c_{0}$ is the concentration of nanoparticles-water suspension on the inlet boundary.

\section{Results and Discussion}

The above highly nonlinear parabolic partial differential equation is solved numerically using an efficient algorithm [31]. The spatial discretization is handled by Galerkin method, while an adaptive time step is used with the time integration. The above governing equations (24), (28), (31), and (32) are solved along with their initial and boundary conditions (40)-(41). The following parameters values are used in the computations; namely [8], $\gamma_{d}=16\left[\mathrm{~m}^{-1}\right], \gamma_{p t}=$ $1.28\left[\mathrm{~m}^{-1}\right], \gamma_{e}=30\left[\mathrm{~m}^{-1}\right], u_{c}=4.6 \times 10^{-6}\left[\mathrm{~m} \cdot \mathrm{s}^{-1}\right]$, and $D=5.6 \times 10^{-8}\left[\mathrm{~m}^{2} \cdot \mathrm{s}^{-1}\right]$. The nanoparticles diameter is taken as $40 \mathrm{~nm}$ and the inlet concentration $c_{0}=0.0009$. Other different values of $c_{0}$ were taken into account in our 


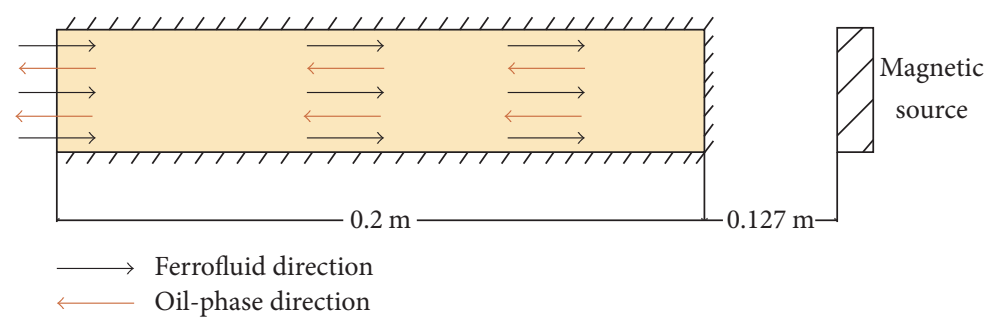

FIGURE 2: Magnet is placed on the right side of the core, with a core length, $h=0.2 \mathrm{~m}$, and magnet parameters, $a_{1}=1.5 \times 10^{4}, b_{1}=$ $2.4 \times 10^{-5}, B r=1.19, a=b=0.0254, L=0.127 \mathrm{~m}$, and $\mu_{0}=4 \pi 10^{-7}$, and magnet positioned at $x=0.2 \mathrm{~m}, y=|x-0.2| \mathrm{m}$.

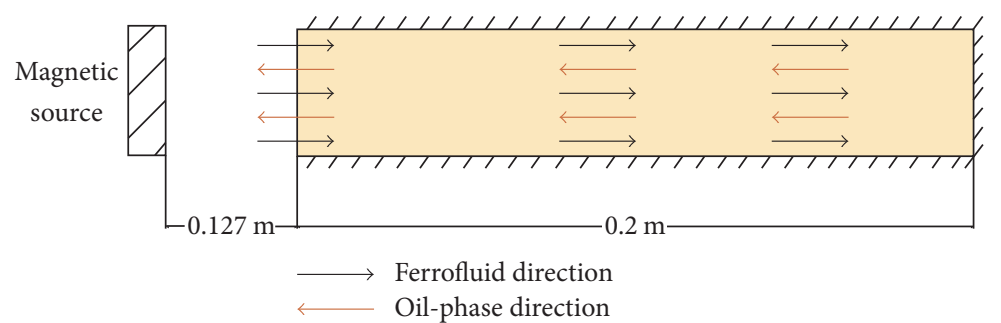

FIGURE 3: Magnet is placed on the left side of the core, with a core length, $h=0.2 \mathrm{~m}$, and magnet parameters, $a_{1}=3 \times 10^{2}, b_{1}=3 \times 10^{-5}, B r=$ $1.19, a=b=0.0254, L=0.127 \mathrm{~m}$, and $\mu_{0}=4 \pi 10^{-7}$, and magnet positioned at $x=0.2 \mathrm{~m}, y=|x-0.2| \mathrm{m}$.

previous study [12]. The remaining model parameters are $[12,32] ; h=0.2 \mathrm{~m}, S_{w r}=S_{o r}=0.1, a_{w}=a_{o}=$ $0.5, \mu_{w}=\mu_{o}=0.001\left[\mathrm{~kg} \mathrm{~m}^{-1} \mathrm{~s}^{-1}\right], \rho_{w}=1000\left[\mathrm{~kg} \cdot \mathrm{m}^{-3}\right], \rho_{o}=$ $660[\mathrm{~kg} \cdot \mathrm{m}], \phi_{0}=0.3, l=3, k_{f}=0.6, \gamma_{f}=0.01, k_{r w}^{0}=$ $k_{r o}^{0}=1, a_{2}=b_{2}=4, \beta=0.8, k_{0}=20 \times 10^{-15}\left[\mathrm{~m}^{2}\right], \theta_{o}=$ 3, $\theta_{w}=0.6$. Moreover, the magnetic field parameters are $a_{1}=1.5 \times 10^{4}, b_{1}=2.4 \times 10^{-5}$ (when the magnet is on the right side of the core). But when the magnet is on the left side of the core, they are taken as $a_{1}=3 \times 10^{2}, b_{1}=3 \times 10^{-5}$.

In the following we consider two different locations of the magnet, namely, right and left side, with respect to the core and its boundary conditions (see Figures 2 and 3). On other words, the magnet location and flow directions are very important factors in this problem. If the magnet location is on the right side of the no-flow boundary of the core, the magnetic field will be assisting the flow, while the opposite is true when the magnet is placed on the left side of the inflow boundary of the rock (opposing flow).

3.1. Magnet at the Right Side of the Core. Firstly, we present the first case in Figures 4-9. Figure 4 illustrates the nanoparticles-water saturation profiles against the rock length with and without magnetic field effect for various values of the time of imbibition. It is interesting to note from this figure the effect of placing the magnet on the right side of the rock as it increases the saturation of nanoparticleswater suspension on this side while it decreases the saturation on the left side of the rock near to the flow inlet. This can be explained through an important fact that is the magnetic force is proportional to magnetic field strength, so fluid that is closer to the magnet is more strongly magnetized and pulled more strongly toward the magnet. For instance, this is in contrast to gravity driven flow in which gravitational force is

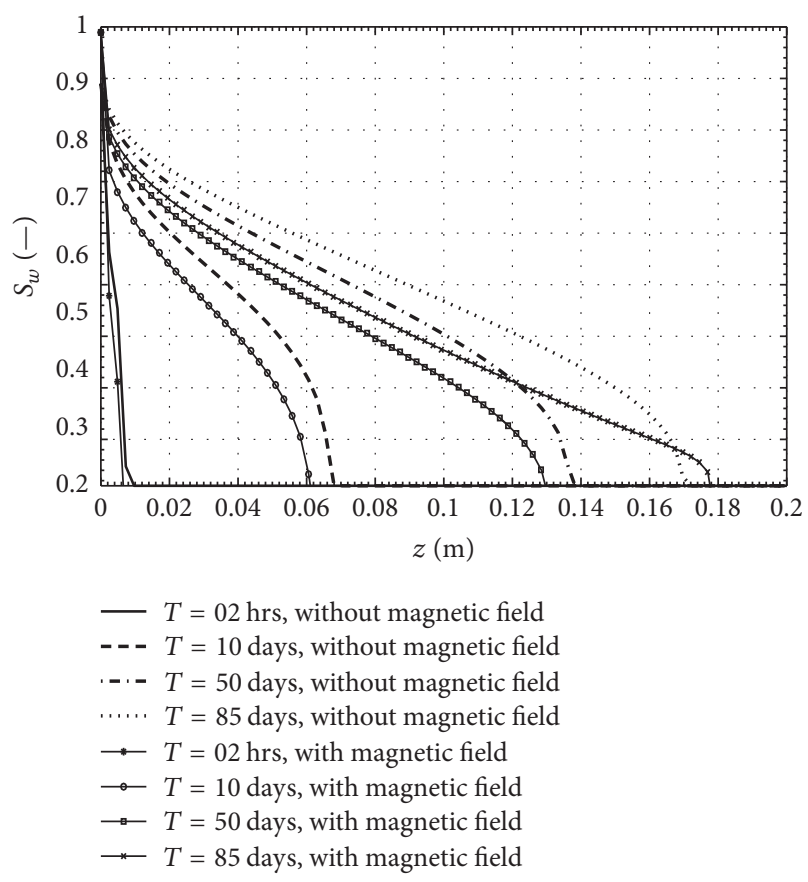

FIGURE 4: Saturations profiles against the core length with and without magnetic field effect for various values of imbibition time: magnet is placed on the right side of the core.

independent of position and fluid is pulled uniformly under gravity.

The nanoparticles concentration is plotted against the rock length in Figure 5, with and without magnetic field effect for various values of the imbibition time. From this figure, it can be seen that after long time of imbibition, 

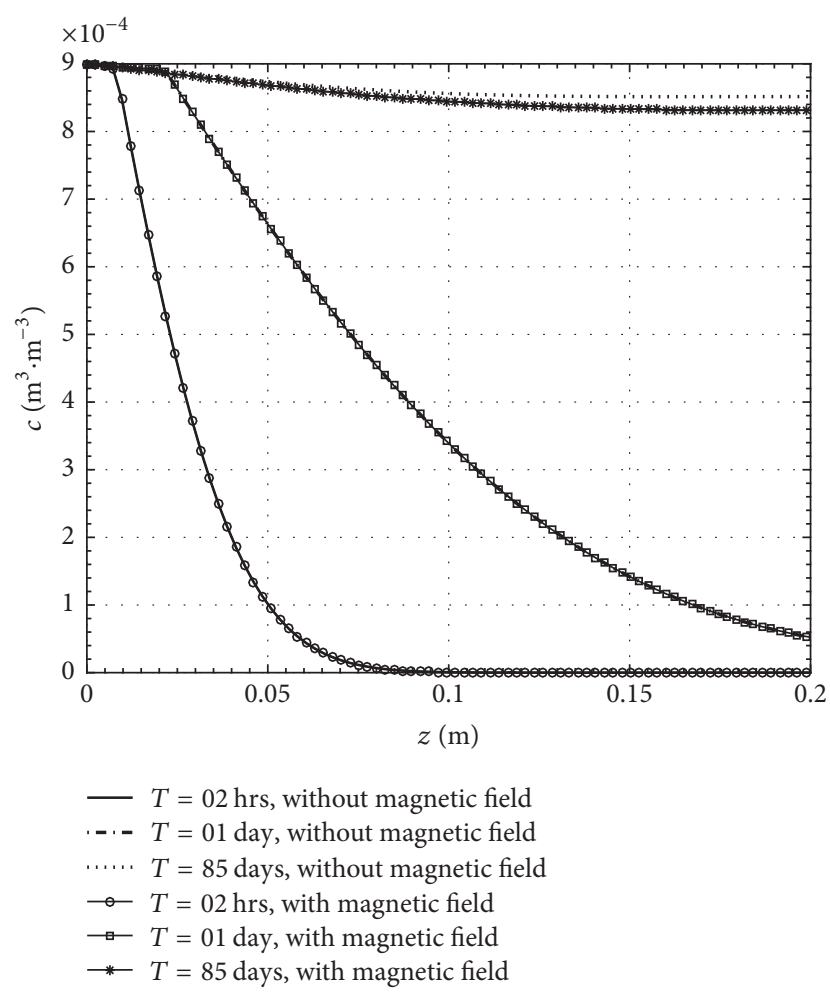

FIGURE 5: Nanoparticles concentration against core length with and without magnetic field effect for various values of imbibition time: magnet is placed on the right side of the core.

the nanoparticles concentration decreases slightly under the effect of the magnetic field. Figure 6 shows the concentration of deposited nanoparticles on the pore wall as plotted against the core length with and without magnetic field effect for various values imbibition times. Also, Figure 7 shows the concentration of deposited nanoparticles on the pore throat which is plotted against the core length with and without magnetic field effect for various values imbibition times. As expected the deposited nanoparticles have an opposite behavior of the nanoparticles in the water; that is, when the nanoparticles concentration in the water decreases, the deposited nanoparticles concentration increases.

In Figure 8, the permeability variation is plotted against the distance, with and without the magnetic field effect for various values of imbibition time when the magnet is located on the right side of the core. It is clear from this figure that the reduction in permeability is related to the quantity of the precipitation of nanoparticles on the wall and the imbibition time. Also, in Figure 9, the porosity variation is plotted against the distance with and without the magnetic field effect for various values of imbibition time when the magnet is located on the right side of the core. It can be seen that the change in porosity is related to the quantity of nanoparticles plugging the throat of the pore and imbibition time.

3.2. Magnet at the Left Side of the Core. Now, we consider the second case, when the magnet is to the left of the rock (which opposes the flow), in Figures 10-13. Saturations

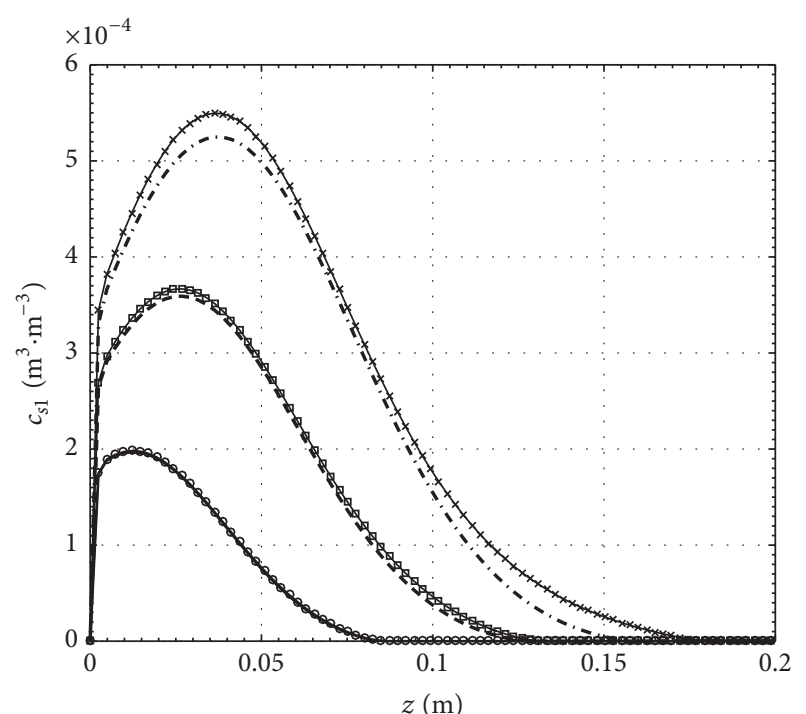

$-T=20$ days, without magnetic field
$--T=50$ days, without magnetic field
$--T=85$ days, without magnetic field
$-T=20$ days, with magnetic field
$-T=50$ days, with magnetic field
$-\leftarrow T=85$ days, with magnetic field

FIGURE 6: Pore wall deposited nanoparticles concentration against core length with and without magnetic field effect for various values of imbibition time: magnet is placed on the right side of the core.

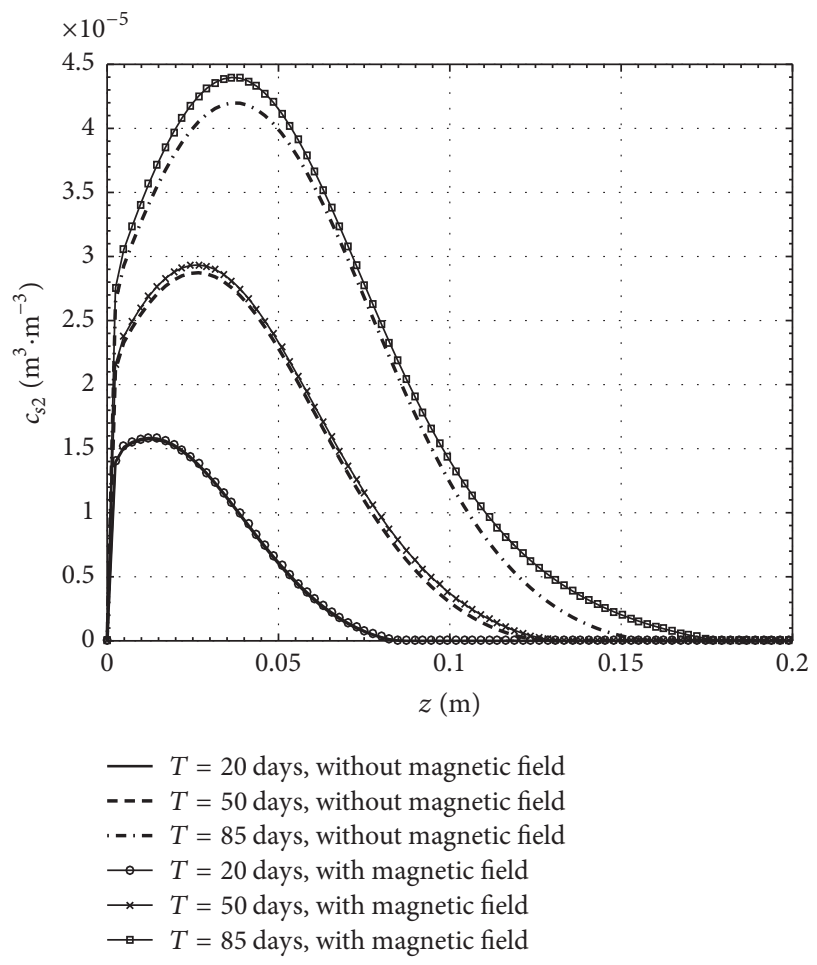

FIGURE 7: Pore throat deposited nanoparticles concentration against core length with and without magnetic field effect for various values of imbibition time: magnet is placed on the right side of the core. 


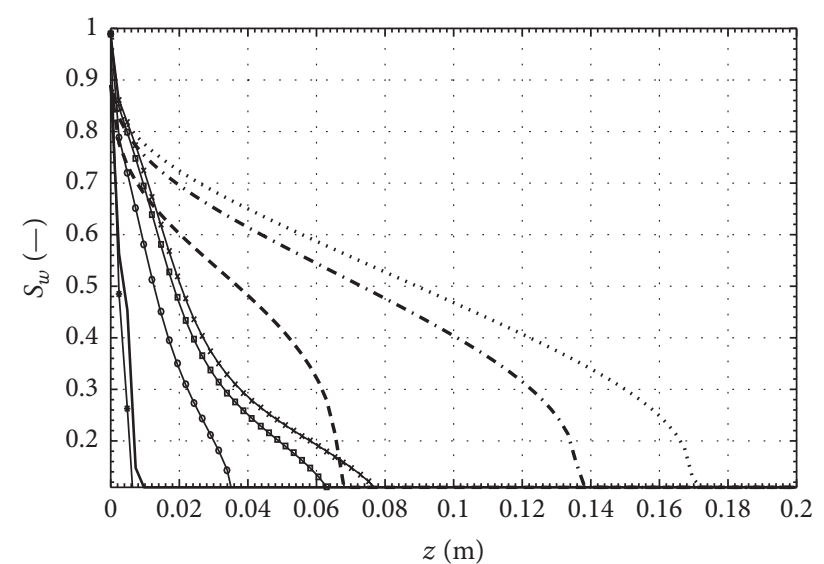

$-T=02$ hrs, without magnetic field
$--T=10$ days, without magnetic field
$\because-T=50$ days, without magnetic field
$\cdots T=85$ days, without magnetic field
$\rightarrow T=02$ hrs, with magnetic field
$\rightarrow T=10$ days, with magnetic field
$\rightarrow T=50$ days, with magnetic field
$\rightarrow T=85$ days, with magnetic field

FIgURE 8: Permeability variation against core length with and without magnetic field effect for various values of imbibition time: magnet is placed on the right side of the core.

profiles are plotted in Figure 10, against the core distance with and without magnetic field effect for various values of imbibition time when the magnet is placed on left side of the core. An interesting behavior can be seen in this figure. Unlike the case of the right magnet, the left magnet resists the flow and reduces water invasion. Moreover, Figure 11 shows the profiles of nanoparticles concentration against core length with and without magnetic field effect for various values of imbibition time, in the case of left location of the magnet. It is clear from this figure that the concentration of nanoparticles in water increases especially after long imbibition time. Figure 12 illustrates deposited nanoparticles concentration on the pore wall against the core length, with and without magnetic field effect for various values of imbibition time when the magnet is located on the left side of the core. This figure shows that the magnetic field reduces significantly the deposited nanoparticles on the pore surface. Finally, Figure 13 illustrates the nanoparticles concentration plugging pore throat against core length with and without magnetic field effect for various values of imbibition time when the magnet is located on the left side of the core. One may notice that the magnetic field reduces the nanoparticles plugging pore throat.

Figure 14 illustrates the permeability profiles against the distance with various values of imbibition time, with and without magnetic field effect when the magnet is on the left side. Similarly, the permeability decreases as a consequence of the precipitation of nanoparticles. The permeability has a minor reduction compared to the initial permeability. Figure 15 shows the porosity variation against the distance with various values of imbibition time, with and without

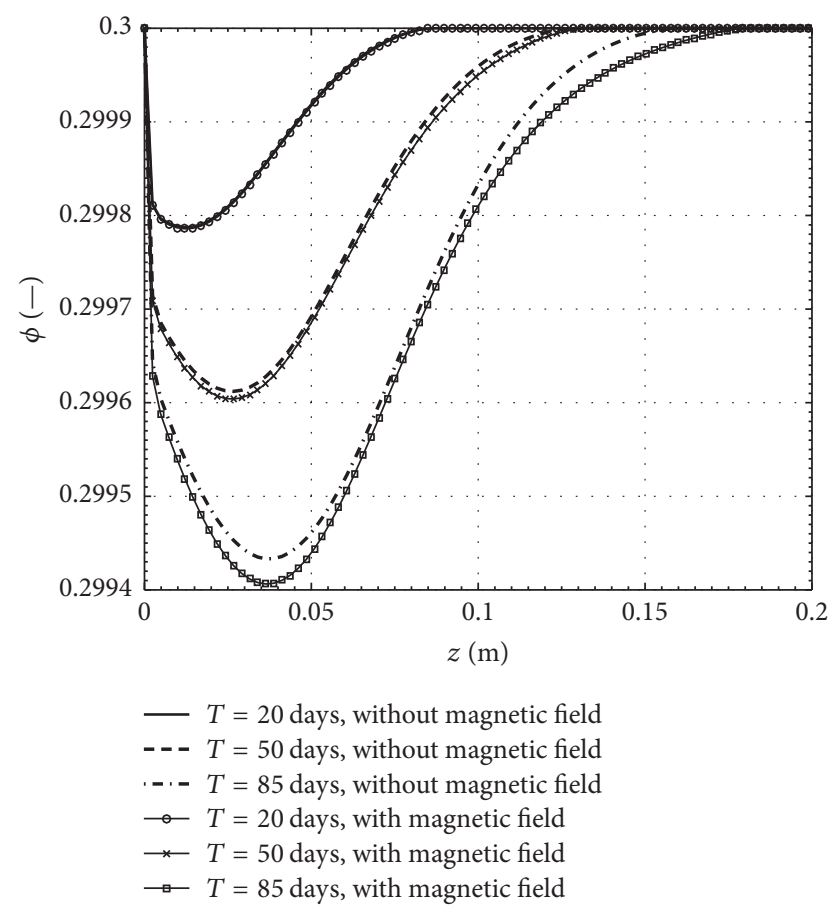

FIGURE 9: Porosity variation against core length with and without magnetic field effect for various values of imbibition time: magnet is placed on the right side of the core.

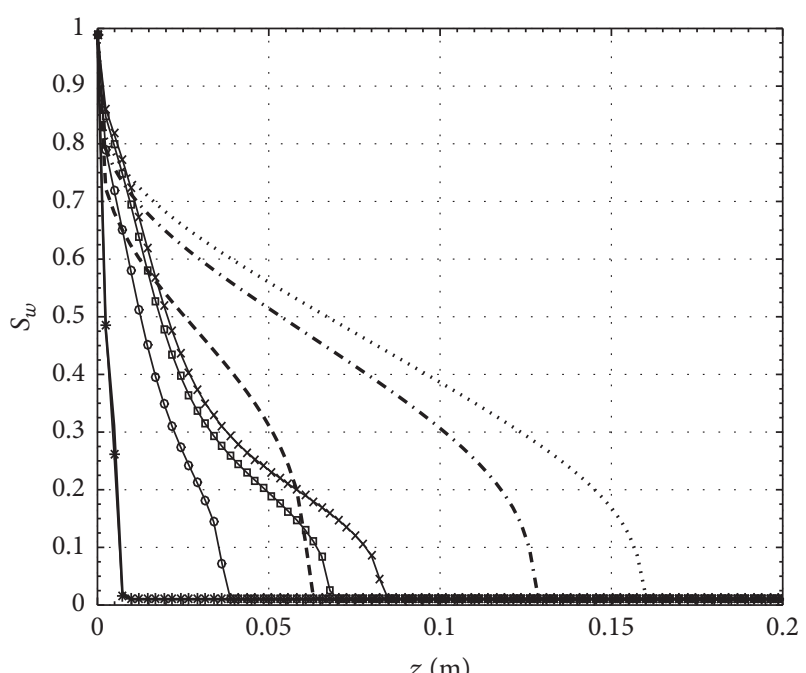

- $T=02 \mathrm{hrs}$, without magnetic field

- - $T=10$ days, without magnetic field

-. - $T=50$ days, without magnetic field

… $T=85$ days, without magnetic field

* $T=02 \mathrm{hrs}$, with magnetic field

$\because T=10$ days, with magnetic field

$\rightarrow T=50$ days, with magnetic field

$* T=85$ days, with magnetic field

FIGURE 10: Saturations profiles against the core length with and without magnetic field effect for various values of imbibition time: magnet is placed on the left side of the core. 


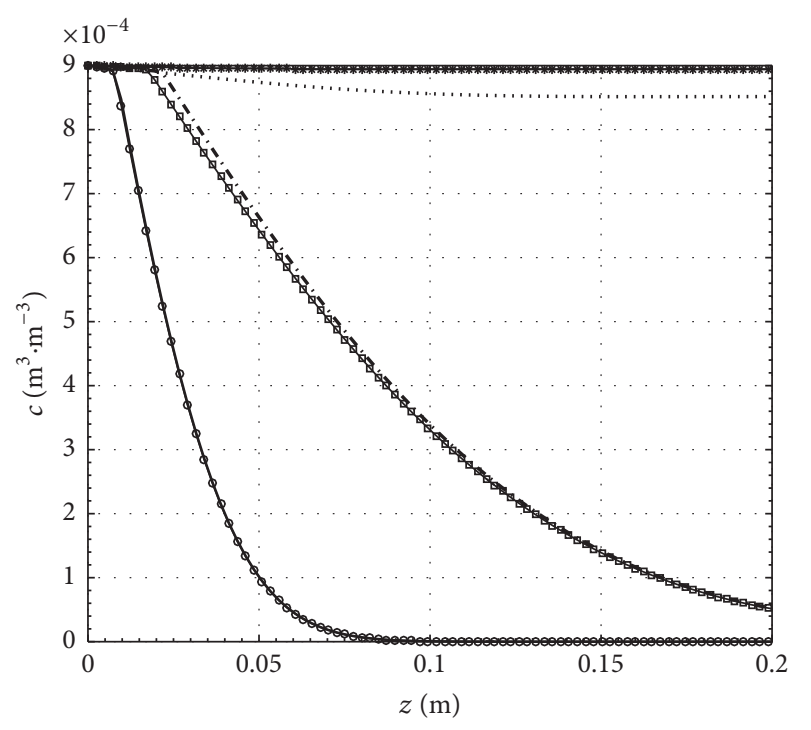

$\longrightarrow T=02$ hrs, without magnetic field
$\cdots-T=01$ day, without magnetic field
$\cdots \cdots T=85$ days, without magnetic field
$\rightarrow-T=02$ hrs, with magnetic field
$\rightarrow-T=01$ day, with magnetic field
$\rightarrow-T=85$ days, with magnetic field

FIGURE 11: Nanoparticles concentration against core length with and without magnetic field effect for various values of imbibition time: magnet is placed on the left side of the core.

magnetic field effect when the magnet is located on the left side. It is interesting to note reduction in the porosity which is a consequence of the precipitation of nanoparticles on the pore walls. The porosity variation is negligibly small.

\section{Conclusions}

This paper was devoted to study the magnetic field effects on the the transport of magnetic nanoparticles injected into a two-phase water-oil system in porous media. Firstly, we developed the mathematical model which governs the transport of magnetic nanoparticles in a two-phase, wateroil system in porous media under the effect of an external magnetic field. The countercurrent imbibition in a smallscale porous medium core is considered as an example. Both Brownian diffusion and mechanical dispersion are taken into consideration, with the assumption that only one size interval of the nanoparticles exists. Variation of porosity, permeability, and relative permeabilities due to particles deposition are also considered. Immiscible mixture relationships have been used to determine the magnetization properties, density, and viscosity of this ferrofluid. In such flow field, the pressure includes additional terms, namely, the ferrofluid dynamic pressure, the fluid magnetic pressure, and the magnetostrictive pressure. Two different locations of the magnetic field with respect to the core inflow and no-flow boundaries are considered. Effects of the magnetic field on the physical variables such as saturation, nanoparticles concentrations, porosity, and permeability have been examined.
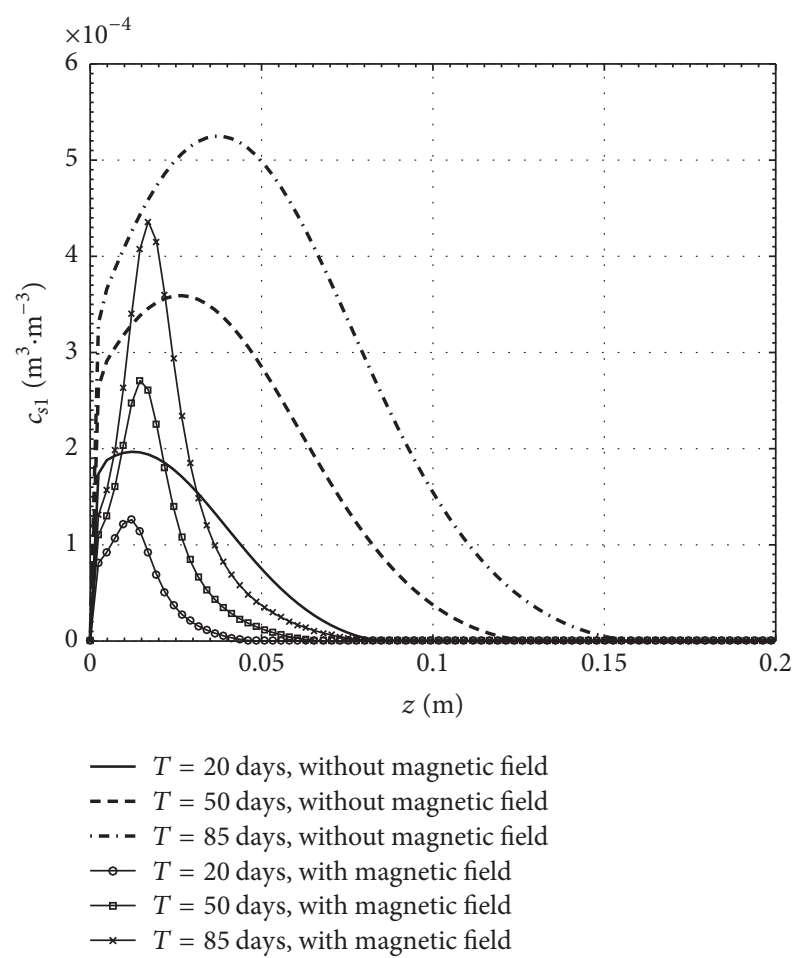

FIGURE 12: Pore wall deposited nanoparticles concentration against core length with and without magnetic field effect for various values of imbibition time: magnet is placed on the left side of the core.

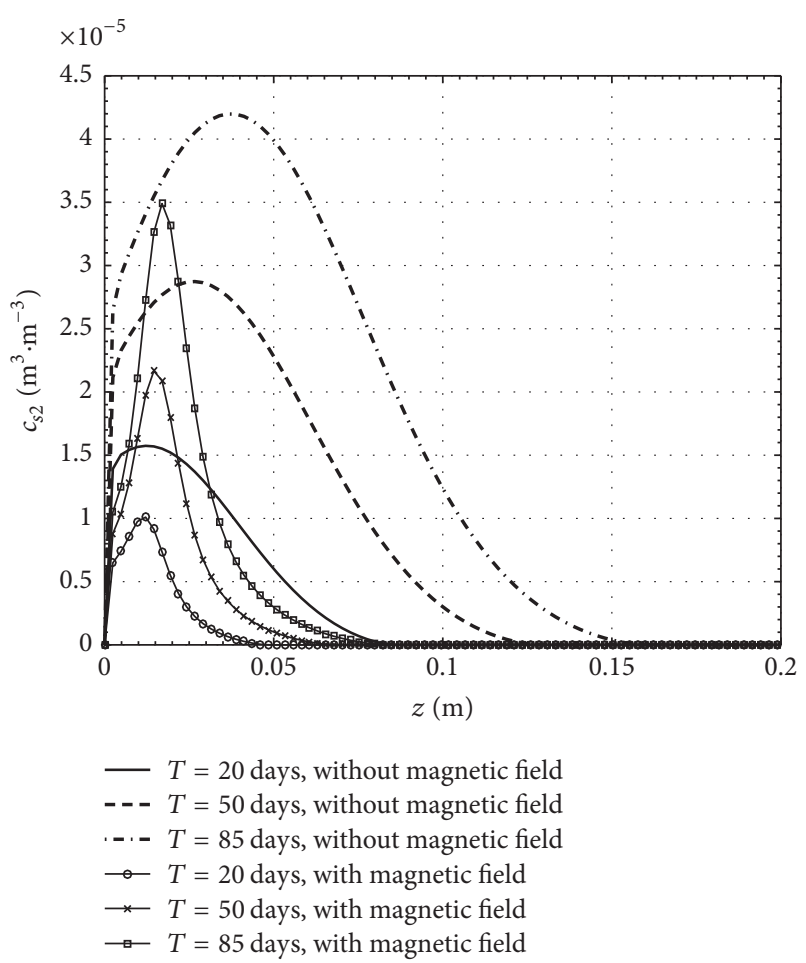

FIGURE 13: Pore throat deposited nanoparticles concentration against core distance with and without magnetic field effect for various values of imbibition time: magnet is placed on the left side of the core. 


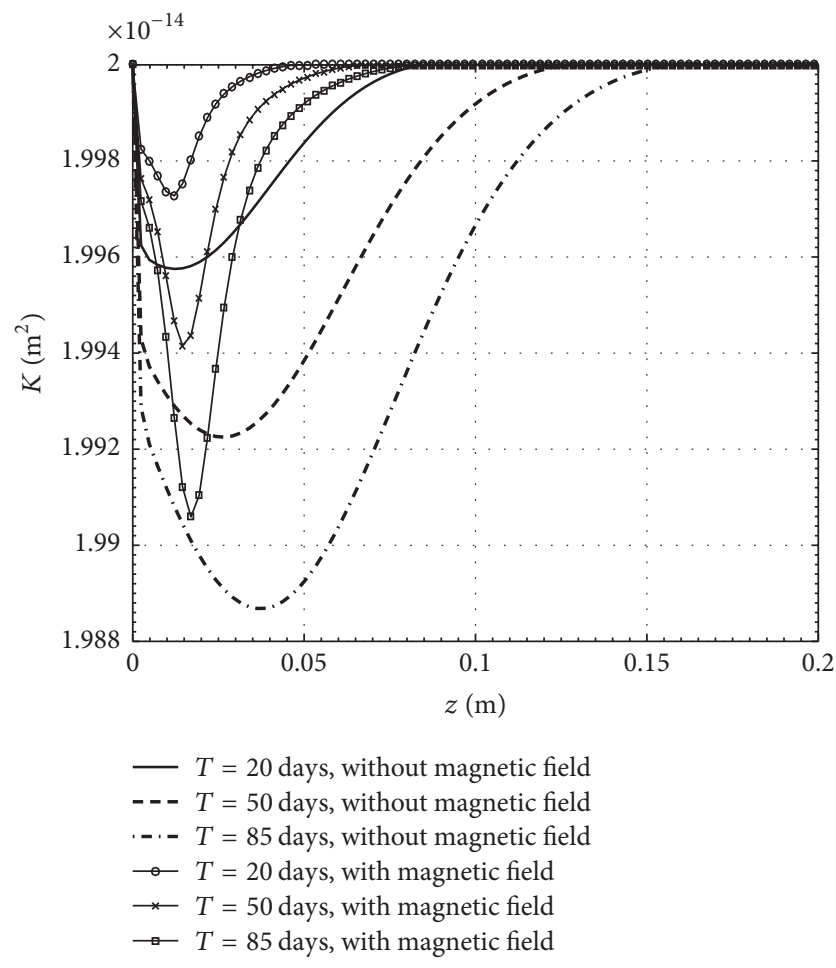

Figure 14: Permeability variation against core length with and without magnetic field effect for various values of imbibition time: magnet is placed on the left side of the core.

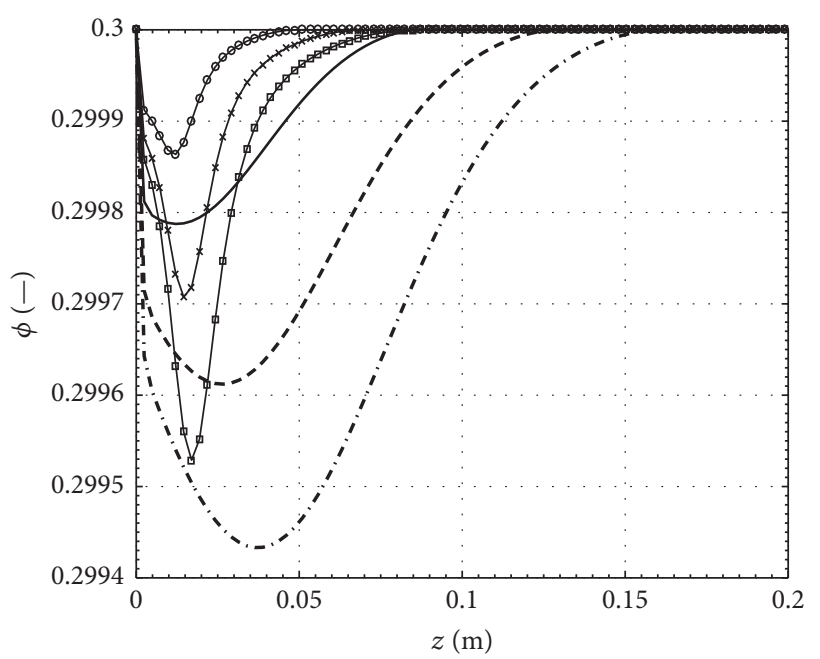

$-T=20$ days, without magnetic field

- - $T=50$ days, without magnetic field

- . - $T=85$ days, without magnetic field

$\rightarrow T=20$ days, with magnetic field

$\star \quad T=50$ days, with magnetic field

$\rightarrow T=85$ days, with magnetic field

FIGURE 15: Porosity variation against core length with and without magnetic field effect for various values of imbibition time: magnet is placed on the left side of the core.
The important result from this simulation is that the magnetic source location has a significant influence on the physical variables of the model. Based on the flow direction and the location of the magnet, the magnetic field can assist or oppose the flow of this two-phase system. Such observed behavior can be used for different applications depending on whether the goal is to aid or delay the injected fluid advancement. According to this investigation, if the magnet is placed next to the no-flow boundary of the studied countercurrent numerical experiment, the saturation of nanoparticleswater suspension increases. Moreover, the concentration of nanoparticles is observed to decrease slightly, which has been attributed to the slight increase of the deposition of nanoparticles. On the other hand, when the magnet is placed next to the inflow/outflow boundary, the magnet resists the flow of the ferrofluid suspension and decreases water invasion capacity, thereby. Furthermore, the nanoparticles concentration seems to increase under the effect of the magnetic field, and the deposited nanoparticles concentration decreases. Both porosity and permeability are reduced due to the nanoparticles adherence to the walls of the porous media.

\section{Nomenclature}

A: $\quad$ Cross-sectional area $\left[\mathrm{m}^{2}\right]$

$a: \quad$ Half of width of the magnet [m]

$a_{1}: \quad$ Constant $\left[\mathrm{A} \mathrm{m}^{-1}\right]$

$a_{\text {tot }}:$ Total surface area in contact with fluids $\left[\mathrm{m}^{2}\right]$

$a_{2}: \quad$ Positive real number [-]

$b$ : $\quad$ Half of height of the magnet $[\mathrm{m}]$

$b_{1}: \quad$ Constant $\left[\mathrm{m} \mathrm{A}^{-1}\right]$

$b_{2}: \quad$ Positive real number $[-]$

$B_{r}: \quad$ Residual magnetization [T]

$c: \quad$ Concentration of nanoparticles in the water phase $\left[\mathrm{m}^{3} \cdot \mathrm{m}^{-3}\right]$

$c_{0}$ : Concentration of nanoparticles in the water on the inlet boundary $\left[\mathrm{m}^{3} \cdot \mathrm{m}^{-3}\right]$

$c_{s 1}$ : Concentration of the deposited nanoparticles on the pore surfaces $\left[\mathrm{m}^{3} \cdot \mathrm{m}^{-3}\right]$

$c_{s 2}$ : Concentration of nanoparticles entrapped in pore throats $\left[\mathrm{m}^{3} \cdot \mathrm{m}^{-3}\right]$

$d$ : Diameter of the nanoparticles in a given interval size [m]

$D_{\text {diff }}$ : Molecular diffusion coefficient $\left[\mathrm{m}^{2} \cdot \mathrm{s}^{-1}\right]$

$D_{\text {disp }}$ : Mechanical dispersion coefficient $\left[\mathrm{m}^{2} \cdot \mathrm{s}^{-1}\right]$

$d_{l, w}:$ Longitudinal dispersion coefficient $[-]$

$f: \quad$ Flow efficiency factor $[-]$

$\mathrm{F}_{\text {mag }}$ : External magnetic force $[\mathrm{N}]$

$f_{\alpha}: \quad$ Flow fraction of the phase $\alpha[-]$

$f_{w}$ : Flow fraction of water [-]

$f_{o}: \quad$ Flow fraction of oil [-]

$g: \quad$ Gravitational acceleration $\left[\mathrm{m} \cdot \mathrm{s}^{-2}\right]$

$H: \quad$ Magnetic field strength $\left[\mathrm{A} \mathrm{m}^{-1}\right]$

$h: \quad$ Rock depth [m]

K: $\quad$ Permeability $\left[\mathrm{m}^{2}\right]$

$K_{0}: \quad$ Initial permeability $\left[\mathrm{m}^{2}\right]$

$k_{f}$ : Constant for fluid seepage

$k_{r \alpha}:$ Relative permeability of the phase $\alpha[-]$ 
$k_{r \alpha, c}:$ Relative permeability of the phase $\alpha$ when the surface is completely occupied by the nanoparticles [-]

$k_{r \alpha}^{0}:$ Endpoint relative permeability of the phase $\alpha[-]$

$k_{B}$ : Boltzmann constant $[-]$

$l: \quad$ Constant [-]

$L: \quad$ Distance between the poles of the magnet $[\mathrm{m}]$

$M$ : Magnetization $\left[\mathrm{A} \mathrm{m}^{-1}\right]$

$p_{\alpha}: \quad$ Pressure of the phase $\alpha[\mathrm{Pa}]$

$p_{w}:$ Ferrofluid phase pressure $[\mathrm{Pa}]$

$p_{o}: \quad$ Oil phase pressure $[\mathrm{Pa}]$

$p_{d}: \quad$ Pressure entry $[\mathrm{Pa}]$

$p_{c}: \quad$ Capillary pressure $[\mathrm{Pa}]$

$p_{m}: \quad$ Fluid magnetic pressure $[\mathrm{Pa}]$

$p_{s}: \quad$ Magnetostrictive pressure $[\mathrm{Pa}]$

$p_{n}: \quad$ Magnetic normal pressure $[\mathrm{Pa}]$

$S: \quad$ Normalized saturation $[-]$

$S_{w}:$ Ferrofluid saturation $[-]$

$S_{w}^{0}: \quad$ Initial ferrofluid saturation $[-]$

$r_{a}: \quad$ Specific area of the sand core $[-]$

$S_{o}: \quad$ Oil saturation $[-]$

$S_{\alpha}$ : Saturation of the phase $\alpha[-]$

$S_{i w}:$ Irreducible water saturation $[-]$

$S_{r o}:$ Residual oil saturation [-]

$t: \quad$ Time [s]

$T: \quad$ Absolute temperature $[\mathrm{K}]$

$V: \quad$ Specific volume $\left[\mathrm{m}^{3} \mathrm{Kg}^{-1}\right]$

$u_{c}: \quad$ Critical velocity $\left[\mathrm{m} \cdot \mathrm{s}^{-1}\right]$

$\mathbf{u}_{\alpha}$ : Velocity of the phase $\alpha\left[\mathrm{m} \cdot \mathrm{s}^{-1}\right]$

$\mathbf{u}_{t}$ : Total velocity $\left[\mathrm{m} \cdot \mathrm{s}^{-1}\right]$

$z: \quad$ Depth $[\mathrm{m}]$.

\section{Greek Symbols}

$\gamma_{d}: \quad$ Rate coefficient for surface retention $\left[\mathrm{m}^{-1}\right]$

$\gamma_{e}: \quad$ Rate coefficient for surface entrainment $\left[\mathrm{m}^{-1}\right]$

$\gamma_{p t}$ : Constant for pore throat blocking $\left[\mathrm{m}^{-1}\right]$

$\gamma_{f}$ : Coefficient of flow efficiency

$\rho_{w, p}:$ Density of water component $\left[\mathrm{kg} \cdot \mathrm{m}^{-3}\right]$

$\rho_{f}:$ Density of ferrofluid component $\left[\mathrm{kg} \cdot \mathrm{m}^{-3}\right]$

$\rho_{\alpha}: \quad$ Density of the phase $\alpha\left[\mathrm{kg} \cdot \mathrm{m}^{-3}\right]$

$\mu_{0}$ : Magnetic permeability $\left[\mathrm{T} \mathrm{m} \mathrm{A}^{-1}\right]$

$\mu_{w, p}:$ Viscosity of pure water $\left[\mathrm{kg} \mathrm{m}^{-1} \mathrm{~s}^{-1}\right]$

$\mu_{\alpha}$ : Viscosity of the phase $\alpha\left[\mathrm{kg} \mathrm{m}^{-1} \mathrm{~s}^{-1}\right]$

$\lambda_{\alpha}$ : Mobility ratio of the phase $\alpha[-]$

$\lambda_{t}:$ Total mobility

$\phi: \quad$ Porosity [-]

$\phi_{0}: \quad$ Initial porosity [-]

$\tau: \quad$ Tortuosity of the flow [-]

$\theta_{\alpha}:$ Ratio of the phase $\alpha$ relative permeability due to nanoparticles adhering $[-]$.

\section{Subscripts and Superscripts}

0 : Reference value

$o$ : The oil phase

$r$ : Residual

$t$ : Total $w$ : The nanoparticles-water suspension phase

$\alpha$ : Phase.

\section{Conflicts of Interest}

The authors declare that they have no conflicts of interest.

\section{References}

[1] S. Kapusta, L. Balzano, and P. M. Te Riele, "Nanotechnology applications in oil and gas exploration and production," in Proceedings of the International Petroleum Technology Conference, Bangkok, Thailand, 2012.

[2] A. Fletcher and J. Davis, "How EOR can be transformed by nanotechnology," in Proceedings of the SPE Improved Oil Recovery Symposium, Tulsa, Okla, USA, 2010.

[3] M. Sabet, S. N. Hosseini, A. Zamani, Z. Hosseini, and H. Soleimani, "Application of nanotechnology for enhanced oil recovery: A review," Defect and Diffusion Forum, vol. 367, pp. 149-156, 2016.

[4] C. Negin, S. Ali, and Q. Xie, "Application of nanotechnology for enhancing oil recovery: a review," Petroleum, vol. 2, no. 4, pp. 324-333, 2016.

[5] M. Cocuzza, C. Pirri, V. Rocca, and F. Verga, "Current and future nanotech applications in the oil industry," American Journal of Applied Sciences, vol. 9, no. 6, pp. 784-793, 2012.

[6] J. J. Sheng, B. Leonhardt, and N. Azri, "Status of polymerflooding technology," Journal of Canadian Petroleum Technology, vol. 54, no. 2, pp. 116-126, 2015.

[7] F. Verga, M. Lombardi, G. Maddinelli, and L. Montanaro, "Introducing core-shell technology for conformance control," Oil \& Gas Science and Technology - Revue d'IFP Energies nouvelles, vol. 72, no. 1, p. 5, 2017.

[8] B. Ju and T. Fan, "Experimental study and mathematical model of nanoparticle transport in porous media," Powder Technology, vol. 192, no. 2, pp. 195-202, 2009.

[9] X. H. Liu and F. Civian, "A multiphase mud fluid infiltration and filter cake formation model," in Proceedings of the SPE International Symposium on Oilfield Chemistry, New Orleans, LA, USA, 1996.

[10] M. F. El-Amin, A. Salama, and S. Sun, "Modeling and simulation of nanoparticles transport in a two-phase flow in porous media," in Proceedings of the International Oilfield Nanotechnology Conference and Exhibition, Society of Petroleum Engineers, Noordwijk, The Netherlands, 2012.

[11] M. F. El-Amin, S. Sun, and A. Salama, "Modeling and simulation of nanoparticle transport in multiphase flows in porous media: CO 2 sequestration," in Proceedings of the Mathematical Methods in Fluid Dynamics and Simulation of Giant Oil and Gas Reservoirs, Society of Petroleum Engineers, Istanbul, Turkey, 2012.

[12] M. F. El-Amin, A. Salama, and S. Sun, "Numerical and dimensional analysis of nanoparticles transport with two-phase flow in porous media," Journal of Petroleum Science and Engineering, vol. 128, pp. 53-64, 2015.

[13] A. Salama, A. Negara, M. El Amin, and S. Sun, "Numerical investigation of nanoparticles transport in anisotropic porous media," Journal of contaminant hydrology, vol. 181, pp. 114-130, 2015.

[14] B. A. Suleimanov, F. S. Ismailov, and E. F. Veliyev, "Nanofluid for enhanced oil recovery," Journal of Petroleum Science and Engineering, vol. 78, no. 2, pp. 431-437, 2011. 
[15] L. Hendraningrat, S. Li, and O. Torsæter, "A coreflood investigation of nanofluid enhanced oil recovery," Journal of Petroleum Science and Engineering, vol. 111, pp. 128-138, 2013.

[16] C. M. Oldenburg, S. E. Borglin, and G. J. Moridis, "Numerical simulation of ferrofluid flow for subsurface environmental engineering applications," Transport in Porous Media, vol. 38, no. 3, pp. 319-344, 2000.

[17] M. Zahn, "Magnetic fluid and nanoparticle applications to nanotechnology," Journal of Nanoparticle Research, vol. 3, no. 1, pp. 73-78, 2001.

[18] N. Yahya, M. Kashif, A. Shafie, H. Solemani, H. M. Zaid, and N. R. A. Latiff, "Improved oil recovery by high magnetic flux density subjected to iron oxide nanofluids," Journal of Nano Research, vol. 26, pp. 89-99, 2014.

[19] N. Kothari, B. Raina, K. B. Chandak, V. Iyer, and H. P. Mahajan, "Application of ferrofluids for enhanced surfactant flooding in IOR," in Proceedings of the SPE SPE EUROPEC/EAGE Annual Conference and Exhibition, Barcelona, Spain, 2010.

[20] S. Ryoo, A. R. Rahmani, K. Y. Yoon et al., "Theoretical and experimental investigation of the motion of multiphase fluids containing paramagnetic nanoparticles in porous media," Journal of Petroleum Science and Engineering, vol. 81, pp. 129-144, 2012.

[21] M. Prodanovic, S. Ryoo, A. R. Rahmani et al., "Effects of magnetic field on the motion of multiphase fluids containing paramagnetic nanoparticles in porous media," in Proceedings of the SPE Improved Oil Recovery Symposium, Tulsa, Okla, USA, 2010.

[22] M. F. El-Amin and T. Brahimi, "Numerical modeling of magnetic nanoparticles transport in a two-phase flow in porous media," in Proceedings of the SPE Reservoir Characterisation and Simulation Conference, Abu Dhabi, UAE, 2017.

[23] M. F. El-Amin, A. M. Saad, S. Sun, and A. Salama, "Numerical simulation of magnetic nanoparticles injection into two-phase flow in a porous medium," in Proceedings of the International Conference on Computational Science, ICCS 2017, vol. 108, pp. 2260-2264, Procedia Computer Science, Zurich, Switzerland, 2017.

[24] S. Borglin, G. Moridis, and A. Becker, "Magnetic detection of ferrofluid injection zones," Lawrence Berkeley National Laboratory Report LBL-40127, Berkeley, Calif, USA, 1998.

[25] M. McCaig and A. G. Clegg, Permanent Magnets in Theory and Practice, Pentech Pres, 2 edition, 1987.

[26] R. E. Rosensweig, Ferrohydrodynamics, Cambridge University Press, 1985.

[27] M. Reeves and R. M. Cranwell, "Theory and implementation of SWIFT II, the Sandia waste-isolation flow and transport model for fractured media," Tech. Rep. SAND83-1159, Sandia National Laboratories, Albuquerque, NM, USA, 1981.

[28] A. W. Herbert, C. P. Jackson, and D. A. Lever, "Coupled groundwater flow and solute transport with fluid density strongly dependent upon concentration," Water Resources Research, vol. 24, no. 10, pp. 1781-1795, 1988.

[29] M. Pooladi-Darvish and A. Firoozabadi, "Co-current and counter-current imbibition in a water-wet matrix block," Society of Petroleum Engineers Journal, vol. 5, no. 1, pp. 3-11, 2000.

[30] C. Gruesbeck and R. E. Collins, "Entrainment and deposition of fines particles in porous media," Society of Petroleum Engineers Journal, vol. 24, pp. 847-856, 1982.

[31] R. D. Skeel and M. Berzins, "A method for the spatial discretization of parabolic equations in one space variable," Society for
Industrial and Applied Mathematics. Journal on Scientific and Statistical Computing, vol. 11, no. 1, pp. 1-32, 1990.

[32] M. F. El-Amin, A. Salama, and S. Sun, "Numerical and dimensional investigation of two-phase countercurrent imbibition in porous media," Journal of Computational and Applied Mathematics, vol. 242, pp. 285-296, 2013. 

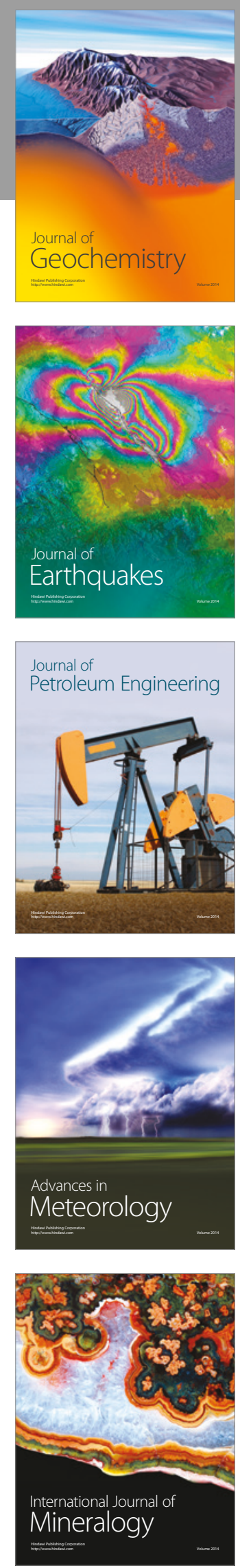
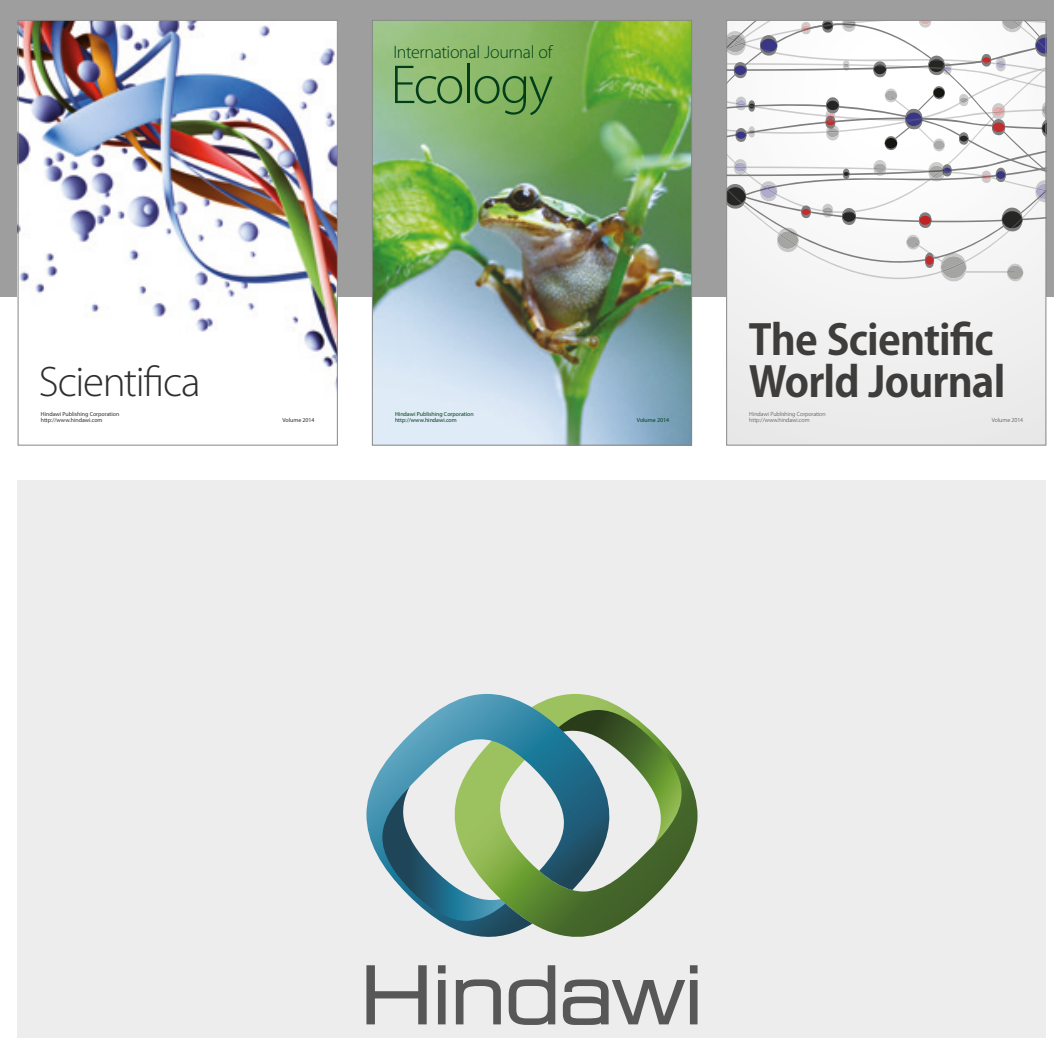

Submit your manuscripts at

https://www.hindawi.com
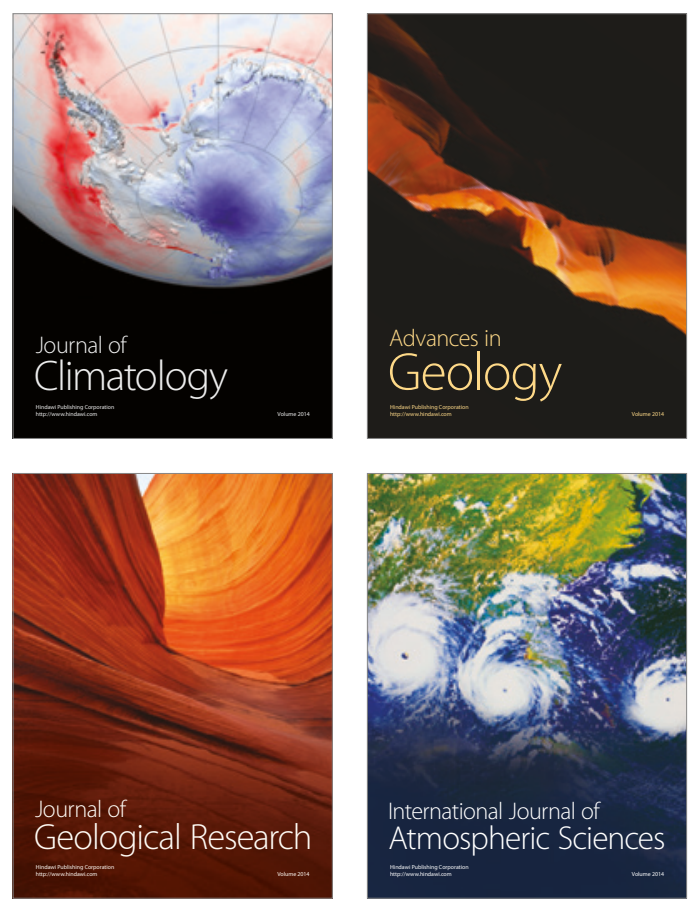

The Scientific

World Journal
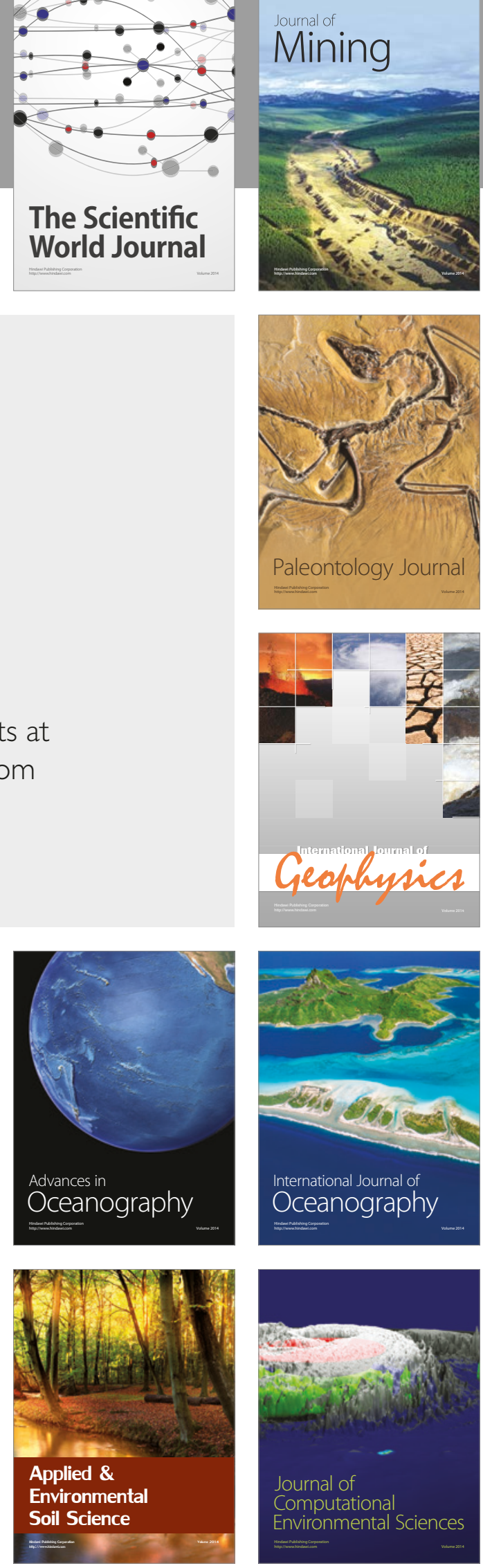